\title{
Caminando hacia la Inclusión a través de la Investigación Acción Participativa en una Comunidad Educativa
}

\section{Walking towards Inclusion through Participatory Action Research in an Educational Community}

\author{
María del Mar Herrera Fernández * \\ Carmen Matés Llamas \\ Diana Farzaneh Peña \\ Sandra Barrado Fernández
}

Colegio de Educación Infantil y Primaria La Parra, España

\begin{abstract}
En el presente artículo se expone la experiencia de un grupo de profesorado con la Investigación Acción Participativa (IAP) en un centro educativo de Andalucía (España) con la implicación activa y protagonista de los miembros de su comunidad educativa como estrategia para la mejora de su propia práctica docente. La investigación surge de una preocupación común de un grupo de docentes: ¿Cómo construir una escuela donde todas las niñas y los niños sin excepción se sientan felices y aprendan? En una primera fase nos preguntamos cómo es la escuela que queríamos y cómo era la escuela que teníamos. Posteriormente, recogimos información de toda la Comunidad para poder decidir en común, qué cuestiones abordar en primer lugar, con la finalidad de diseñar estrategias que, llevadas a la acción, nos permitieran construir esa escuela querida y soñada. Las fases que seguimos durante la investigación fueron la observación, el diagnóstico, la planificación (elaboración de un Plan de Acción Integral), la acción y la evaluación del proceso. Las herramientas que utilizamos durante el proceso han sido la observación participante, las entrevistas abiertas o los sociogramas, entre otras. Los resultados obtenidos nos permiten concluir que la experiencia en IAP nos ha permitido mejorar nuestra escuela.
\end{abstract}

Descriptores: Formación docente; Investigación acción; Innovación educativa; Inclusión educativa; Desarrollo profesional.

This article presents the experience of a group of teachers with Participatory Action Research (PAR) in an educational center in Andalusia (Spain). Their strategy is to improve their own teaching. With this purpose in mind, they propose a more active and leading involvement by the members of their education community. The research arises from a common concern of a group of teachers: How to build a school where all girls and boys without exceptions could be happy and could learn? We started, as a first step, asking ourselves what the school we wanted is like and what the school we had was like. Subsequently, we collected information from the entire Community so that we all can decide which issues to address first, in order to design strategies that, brought into action, would allow us to build that beloved and dreamed school. The steps followed during the investigation were observation, diagnosis, planning (elaboration of a Comprehensive Action Plan), action and evaluation of the process. The tools we used during the process were participant observation, open interviews or sociograms, among others. The results obtained allow us to conclude that the IAP experience has allowed us to improve our school.

Keywords: Teacher education; Research action; Educational innovation; Inclusion educational; Professional development.

*Contacto: meryherrera76@gmail.com

ISSN: 0718-7378

www.rinace.net/rlei/
Recibido: $\quad 30 / 06 / 2021$

$1^{a}$ Evaluación: 09/09/2021

Aceptado: 10/10/2021 


\section{Introducción: Camino a la inclusión}

Caminante, son tus huellas el camino y nada más;
Caminante, no hay camino, se hace camino al andar.
Al andar se hace el camino, y al volver la vista atrás
se ve la senda que nunca se ha de volver a pisar.
Caminante no hay camino sino estelas en la mar. (Machado, 1991, p.93)

Consideramos que todo proceso educativo debe comenzar con el conocimiento de todos sus integrantes, por lo que nos disponemos a hacerlo. Las personas que decidimos emprender este camino somos un grupo de profesorado de un pequeño colegio de la Axarquía malagueña a quienes nos une la ilusión de que el alumnado aprenda entre sí, independientemente de las distintas capacidades que tengan, y que ese aprendizaje se dé conjuntamente dentro del aula. Por otro lado, las personas que decidimos contar esta experiencia en este artículo, tenemos identidades docentes muy distintas: docentes de primaria e infantil con perfil de dirección, docente del Proyecto Roma y pedagogía inclusiva, y Educadora Social con funciones en el colegio como Personal Técnico de Integración Social.

Nuestro colegio ha ido cambiando y transformándose y desde el curso 2015/16 es un centro educativo Comunidad de Aprendizaje que, como lo definen Díez-Palomar y Flecha (2010, p. 25), "es un proyecto global de transformación educativa y social que implica e impacta a muchos agentes, sistemas y procesos sociales".

Las principales pretensiones del Proyecto Educativo de nuestra escuela son conseguir la inclusión educativa y la transformación social, y esta necesidad y voluntad nos lleva al comienzo de todo: cómo construir una escuela inclusiva, donde todas las niñas y todos los niños aprendan.

Comprometerse con una perspectiva inclusiva implica asumir la tarea de pensar y recoger
evidencias que nos permitan responder a cuestiones tales como hasta qué punto la cultura del
centro (...), la forma de organizarnos (...) o el currículum escolar (...) se configuran o no,
como facilitadores del aprendizaje y el rendimiento de todo el alumnado, tal y como están
concebidos y concretados en la actualidad. (Echeita, 2016, p. 10)

Este camino que hemos emprendido no ha estado falto de dificultades y de resistencias. La mayor resistencia la encontramos en que no todo el profesorado comparte el principio de que todo el alumnado puede aprender y de que ha de hacerlo en compañía de sus iguales. Existe la idea de que hay alumnado que no puede aprender, y menos aún dentro de clase, sino que necesita de "otra educación" para poder hacerlo, de "otros profesionales", de "otro currículum" y de un espacio individualizado donde pueda progresar. Se piensa que no se puede construir una escuela donde todas las niñas y niños aprendan juntos, "porque siempre las cosas han sido asî" (entrevista a profesorado de Primaria) o porque no tienen estrategias, ni formación para dar respuesta a las demandas que cada alumno y alumna requiere en el aula.

Una parte del profesorado empezamos a tomar conciencia de cómo se sentía el alumnado al salir del aula con otra docente y no poder aprender con su grupo, del peso de las etiquetas, y también de que el aprendizaje es mayor y más enriquecedor para todos y todas cuando se hace en sociedad y no en soledad con un adulto. Nos planteamos si era posible construir una escuela donde el alumnado con más peculiaridades no tuviera que salir del grupo para aprender, donde los alumnos y alumnas no se sintieran señalados, una escuela donde todos y todas disfrutasen del proceso de aprendizaje juntos. Y para conseguir esto, teníamos claro que era necesario buscar y saber encontrar cuáles eran esas barreras que impedían la presencia y, por tanto, el aprendizaje y la participación de todo el alumnado, cuestiones que, como explica López Melero (2008) son fundamentales plantear en la escuela pública. 
De esta manera el grupo de profesorado que conformamos esta investigación va tomando conciencia de que para hablar de educación inclusiva hay que estar abiertos a cambiar las prácticas pedagógicas, para que estas cada vez sean más humanizadoras y menos segregadoras.

\begin{abstract}
Cambiar las prácticas pedagógicas significa que la mentalidad del profesorado ha de cambiar respecto a las competencias cognitivas y culturales de las personas diferentes, que han de cambiar los sistemas de enseñanza y aprendizaje, el currículum escolar, la organización escolar y los sistemas de evaluación. (López Melero, 2008, p. 4)
\end{abstract}

Casi de la mano de lo anteriormente planteado, vemos que para poder luchar por la escuela que queremos necesitamos ampliar y mejorar la formación docente con el objetivo de, ser capaces de acometer el reto de educar a todo el alumnado junto con toda singularidad que le caracteriza, en definitiva, asumir el reto de ser capaces, como docentes, de procurar justicia social (Zeichner, 2010), y no continuar con la práctica habitual y segregadora donde el alumnado mayoritario aprende en su aula con un docente y el alumnado con más peculiaridades sale de ésta para aprender en compañía de la maestra de pedagogía terapéutica (PT). Y es llegado a este punto cuando comienzan a surgirnos una serie de dudas: qué estrategias utilizar en el aula, si sería posible la docencia con dos docentes a la vez, cómo realizar la coordinación docente, cómo abordar el currículum, cómo construir situaciones de aprendizaje para todo el alumnado y sus familias, etc.

La preocupación común del profesorado de construir una escuela sin exclusiones y la necesidad de formación para poder hacerla posible, nos lleva a un grupo de docentes, entre otras muchas, a la demanda de formación docente a un grupo de profesoras y profesores de la Universidad de Málaga (UMA) que tienen un recorrido en la investigación sobre la inclusión educativa. De dicha formación se nos brindó la oportunidad de participar en una Investigación-Acción-Participativa, dentro de "Narrativas emergentes sobre la escuela inclusiva desde el modelo social de la discapacidad. Resistencia, resiliencia y cambio social" (RTI2018-099218-A-Io0) que dirigen los profesores D. Ignacio Calderón Almendros y $\mathrm{M}^{\mathrm{a}}$ Teresa Rascón Gómez, pertenecientes al área de Teoría e Historia de la Educación de la Universidad de Málaga.

Teníamos, por tanto, la oportunidad de participar e investigar en la escuela que queremos construir entre todos y todas. Para ello, comenzamos planteándonos cuatro cuestiones: ¿Cómo es la escuela que tenemos?, ¿Cómo se enseña en nuestro colegio?, ¿Cómo es la relación alumnado-profesorado?, ¿Cómo es la escuela de tus sueños? Siendo conscientes que lo que pretendíamos no dependía sólo del profesorado, y teniendo presente que la Investigación Acción se basa en el estudio de la realidad educativa (Elliot, 1993) invitamos a la Comunidad al completo a que respondiera a estas preguntas y trabajásemos conjuntamente en dicha construcción porque "analizar la realidad, implica intervenir sobre ella, para conocerla, estudiarla, y mejorarla, lo que supone planificar determinadas pautas, que se verán reflejadas en forma de estudio o investigación, que tendrán como objetivo la obtención de conocimientos" (López Melero, 2011, p. 340) Éste fue el inicio de la creación del Grupo de Investigación Acción Participativa (GIAP) en nuestra comunidad educativa.

Para nosotras, esta investigación ha supuesto un cambio de paradigma, una apertura a la comunidad y una nueva forma de entender la formación y la práctica docente. Nos planteamos la necesidad de cooperar junto a la Comunidad, como explica Ignacio Calderón Almendros (2019a, p. 19), "las escuelas deben abrir sus puertas a la comunidad, creando canales de participación efectiva que puedan revolucionar lo que hoy son prácticas habituales". 


\section{Revisión de la literatura: Por qué caminamos} Enseñar no es transferir conocimiento,
sino crear las posibilidades para su
propia producción o construcción.

(Freire, 1997, p. 47)

Sin duda, lo que nos mueve es la necesidad de ser mejores docentes para poder transformar los contextos educativos y sociales de nuestro alumnado, partiendo de la idea de que "la educación constituye un instrumento indispensable para que la humanidad pueda progresar hacia los ideales de paz, libertad y justicia social” (Delors, 1996, p. 13).

Nuestro objetivo principal con esta investigación es el deseo de transformar nuestro centro, reflexionando y planteándonos nuestra labor educativa, dando respuesta a cada una de las necesidades e inquietudes que presenta el alumnado, donde desaparezcan las prácticas segregadoras, se produzca un cambio de mirada del profesorado y donde todas las personas que conforman la Comunidad educativa participen. "Queremos que los niños y niñas vengan felices al colegio a aprender" (entrevista a una familia)

Esta pretensión nos llevó a tomar conciencia que son muy necesarias las voces de todas las personas que constituyen nuestra comunidad para construir la escuela que queremos dando principalmente la voz a los niños y niñas que han sido la luz en esta andadura, no olvidando que son ellos y ellas los principales protagonistas en esta investigación y los que, en muchas ocasiones, nos muestran el camino la mejora de nuestra labor docente. No hay que olvidar que los fundamentos de la Investigación Acción Participativa "se sostienen desde la manifestación de sus protagonistas, de profundizar en su práctica cotidiana, a partir de sus creencias y planteamientos críticos, lo que les permite generar una transformación en lo personal y en lo social" (López Melero, 2011, p. 353)

Se producen cambios en el papel del profesorado, de forma que deja de concebirse como un mero técnico que reproduce conocimientos, y se convierte en investigador de su propia práctica, reflexionando sobre su labor docente y obteniendo conclusiones que le permitirán construir nuevas situaciones de aprendizaje (Latorre, 2003). Desde esta posición, el profesor también aprende, porque como explicaba Freire (1994), "el educador ya no es solo el que educa sino aquel que, en tanto que educa, es educado a través del diálogo con el educando, quien, al ser educado, también educa” (p. 9)

Cuando ocurre esto, también el papel del alumnado varía, pasando de ser un agente pasivo a ser el sujeto protagonista de su propio aprendizaje. En esta situación, el rol de las familias también adquiere mayor protagonismo, pasando a convertirse en sujetos de cambio y transformación.

\section{Método: Comenzando a construir el camino}

\subsection{La Investigación-Acción Participativa (IAP), una forma de entender la formación permanente del profesorado}

En este nuevo papel del profesorado como docentes investigadores, la Investigación Acción se convierte en la herramienta clave de formación permanente del profesorado. Como describen Kemmis y McTaggart (1988):

La Investigación Acción es un proceso sistemático de aprendizaje en el que las personas actúan conscientemente sin dejar, por ello, de abrirse a la posibilidad de sorpresas y conservando la posibilidad de responder a las oportunidades. Se trata de un proceso de utilización de la 


\begin{abstract}
"inteligencia crítica" orientado a dar forma a nuestra acción y a desarrollarla de tal modo que nuestra acción educativa se convierta en una praxis (acción críticamente informada y comprometida) a través de la cual podamos vivir consecuentemente nuestros valores educativos. (p. 31)
\end{abstract}

La IAP es una alternativa metodológica que enriquece los contextos, pues permite la expansión del conocimiento a la par que "genera respuestas concretas a problemáticas que se plantean los investigadores y coinvestigadores cuando deciden abordar una interrogante, temática de interés o situación problemática y desean aportar alguna alternativa de cambio o transformación" (Colmenares Escalona, 2012, pp. 103-104). Las Investigación Acción se desarrolla en varias fases que son la Planificación, la Acción, la Observación y la Reflexión. Esta última nos permite retomar el proceso de planificación, "en una espiral que no se acaba nunca" (Kemmis y McTaggart, 1998, p. 30).

Como explicamos en el epígrafe anterior, es la necesidad de un cambio en nuestra labor docente la que nos hace iniciar una búsqueda y la que nos lleva a la toma de conciencia de que necesitábamos formación como punto clave para la transformación. Para ello nos ponemos en contacto con la Universidad de Málaga (UMA), a través del CEP de la Axarquía, con el propósito de solicitar formación en inclusión al profesor Ignacio Calderón Almendros, y a las profesoras Teresa Rascón Gómez y Mariana Alonso Briales, del Departamento de Teoría e Historia de la Educación y Métodos de Investigación y Diagnóstico en Educación, por su trayectoria en inclusión educativa.

Durante esas jornadas de formación desmontamos muchas de las falsas creencias, que como docentes habíamos construido y empezamos a tomar conciencia de cómo era la escuela que teníamos, y a soñar con la escuela que queríamos. Al ser un centro de Comunidades de Aprendizaje, esas jornadas formativas contaban con la asistencia del $100 \%$ del claustro de profesoras y profesores. Se ocasionó, por tanto, un encuentro para poder escuchar los distintos puntos de vistas del profesorado que trabajamos juntos, cuáles eran los ideales que perseguíamos y la concepción sobre la escuela que deseábamos y la reflexión de cómo llegar a ella. En ese momento, comenzamos a tomar conciencia de que no todo el equipo de profesionales tenía la misma visión, y que lo que para algunos era evidente, para otros era impensable. A raíz de este proceso de formación, y como posible respuesta a todos los interrogantes y deseos que nos planteábamos, se nos brindó la oportunidad formar parte de un grupo de Investigación Acción con la finalidad de seguir transformando nuestra escuela en una escuela inclusiva. Como explica Calderón Almendros (2019b):

Somos muchos los docentes y las familias que queremos que nuestras escuelas cambien. Necesitamos que se transformen en espacios para todos los niños y las niñas, en los que se cuestione la normalidad. $\Upsilon$ eso no va a ocurrir por arte de magia, sino por nuestra acción decidida. (p. 1)

La participación en dicha investigación, se planteó a todo el claustro de profesorado vinculándose aquellos docentes que de manera voluntaria se sentían en la necesidad de provocar ese cambio. Era de suma importancia, que la vinculación a dicho proyecto partiese de la necesidad que cada uno como profesional sentía y no podía entenderse como un proceso implantado de manera obligatoria. Y pasamos a la acción, ¿cómo empezamos? Lo primero que nos planteamos fue escuchar a toda la Comunidad Educativa para conocer cuáles eran las dificultades que encontraban y la escuela que deseaban para sus hijos e hijas. Se construyó así una relación horizontal entre todos los miembros de la Comunidad Educativa dialogando en asamblea, ya que esta herramienta aporta "a los participantes en el proceso, una vía de reflexión y acción que les convierte en sujetos de sus propias vidas" (Pereda y Prada, 2014, p. 60). Para ello, hicimos coincidir dicho encuentro con la fase del sueño, estrategia propia del proyecto de Comunidades 
de Aprendizaje donde se invita a todos los agentes de la comunidad educativa a que expresen cuál es la escuela que desean tener. La organización del encuentro se realizó en el seno del grupo de investigación. El objetivo era recoger la información en dos de las tres vertientes posibles: alumnado y comunidad educativa en general, exceptuando al profesorado, que pospusimos para más adelante. Se decidió que los grupos de alumnado debían ser heterogéneos, es decir con representación de todas las edades en todos los grupos y asegurar la participación del 100\% del alumnado. Para dinamizar y recabar la información que necesitábamos para nuestra investigación, contamos con la participación de profesorado junto con voluntariado del grado de Pedagogía de la UMA. En dicha jornada se le plantearon a toda la comunidad 4 cuestiones:

- ¿Cómo es la escuela que tenemos? (Panorámica general)

- ¿Cómo se enseña y cómo se aprende en nuestra escuela? (Detección de las barreras aprendizaje)

- ¿Cómo es la relación con tus compañeros/as y profesores/as? (Detección de las dificultades en la convivencia)

\section{Figura 1}

\section{Jornada de fase del sueño}
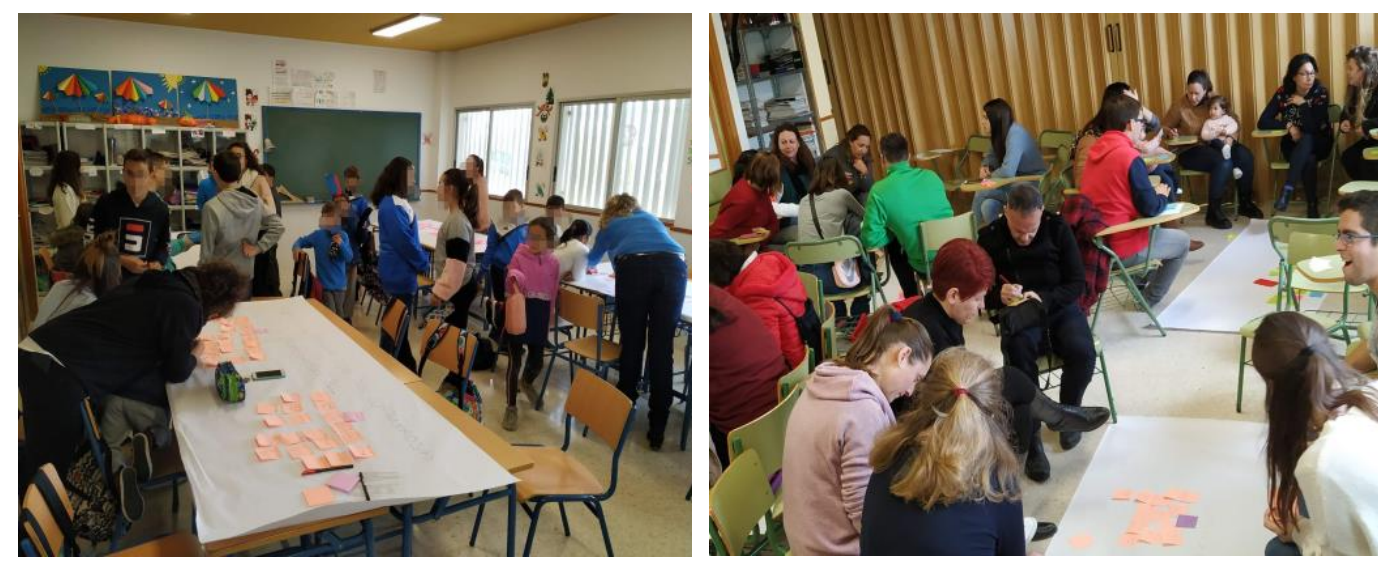

¿Cómo es la escuela de tus sueños? (Proyección de la escuela deseada)

Toda la información recopilada en los distintos grupos, fue analizada por un equipo formado por portavoces de cada subgrupo donde había representación de familias y alumnado, y donde se buscaba el debate y la reflexión sobre aquellos aspectos que se habían estado trabajando. La jornada terminó con una asamblea en la cual participaron todos los sectores de la comunidad, exponiendo, los representantes de cada grupo que habían analizado la información, una síntesis de aquello sobre lo que habían trabajado para así poner en común las dificultades, las fortalezas y los sueños que habían surgido. Es aquí donde emerge un conflicto por una preocupación conjunta relacionada con las relaciones entre el alumnado. Por las exposiciones realizadas y el modo en el que se expresaron, la asamblea desembocó en desavenencias entre todos los sectores participantes. Había miembros del grupo de las familias y del alumnado que se sentían representados en esas intervenciones, pero había otras que no. Cierto profesorado llegó a considerar que el problema radicaba en haber abierto las puertas del centro para que las familias hablaran. Aunque en esos momentos no éramos conscientes de la importancia que tenía este hecho que vivíamos, con el tiempo entendimos que este conflicto generado tras el diálogo era el impulso que necesitábamos para comenzar a producir un cambio. 
El alumnado puso de manifiesto que existían una serie de problemas de convivencia, que dificultaban su aprendizaje. Todas estas reflexiones nos llevaron a plantearnos con mayor claridad la necesidad de indagar en nuestro contexto educativo y en su entorno, para transformarlo, vislumbrando como un posible foco, en esos inicios, la convivencia.

\subsection{Analizando la realidad de la que partimos: Diagnóstico de las relaciones y la convivencia}

La necesidad de analizar más profundamente las relaciones en el centro educativo y su entorno para poder transformar la convivencia fue nuestro motor de investigación. Para poder trabajar de forma comunitaria e integral se configuró un Grupo de Investigación Acción Participativa (GIAP) que se encargó de indagar, junto con el resto de la comunidad educativa, sobre cómo se establecían las relaciones en el contexto y qué necesidades se detectaban. Para dicha investigación, se utilizaron instrumentos como: en la fase de planificación, las entrevistas abiertas; en la fase de diagnóstico, los sociogramas o las observaciones participantes, y el flujograma, tal y como se explica a continuación.

También se ha de destacar que la comunidad educativa ha sido partícipe y protagonista en todo momento, de forma que han participado tanto en la recogida de información como en el análisis de ésta, así como en la realización de propuestas para el Plan de Acción Integral.

\subsection{Constitución del Grupo de Investigación Acción Participativa y análisis de la información}

Una vez realizadas todas las preguntas a todos los miembros de la comunidad educativa y recogidas todas las aportaciones en papel, en cada una de las cuatro cuestiones planteadas, debíamos ahora analizarlas con el fin de obtener cuáles son las líneas de transformación que la comunidad educativa nos sugería, los puntos sobre los que debemos intervenir, trabajar y reflexionar sobre cómo construir una escuela para todos y todas.

Para llevar a cabo dicho análisis, era necesario la constitución de un grupo motor de trabajo, un Grupo de Investigación Acción Participativa (GIAP) formado por los docentes del centro que de manera voluntaria ya habían expresado su implicación con dicha investigación; alumnado, familias, personal de administración y servicios, otros agentes de la comunidad; y profesorado, alumnado e investigadores de la Universidad de Málaga. Éramos conscientes que, cuanta mayor representación hubiera en el grupo GIAP, más ricas iban a ser las aportaciones, las reflexiones y el análisis que en su seno se produjeran, la representación de sectores debía estar equilibrada. La constitución de dicho grupo se llevó a cabo fijando cuáles eran las bases del mismo, estableciendo una jerarquía horizontal, donde la opinión y la voz de cada uno de los integrantes tenían el mismo peso o valor. Se distribuyeron distintos roles dentro del grupo para dinamizarlo y se marcó una premisa que consideramos fundamental en todo el camino que teníamos por delante: disfrutar de todo el proceso que estábamos viviendo. Marcamos por tanto un calendario sistemático de reuniones para trabajar. Hay que señalar que, el profesorado integrante del GIAP ya conocía la investigación, pero el resto de participantes de los distintos sectores, sobre todo el alumnado, no lo tuvieron claro hasta que se abordó en la primera sesión de constitución del grupo. Por ello, cabe destacar la actitud, motivación, deseo, constancia, de los integrantes que ha sido un factor fundamental para que el grupo funcionara.

Lo primero que se realizó una vez formado el GIAP fue recopilar en un único documento todas las aportaciones que teníamos en los distintos formatos. Una vez elaborado dicho documento, realizado con la ayuda del equipo de la UMA, abordamos el primer bloque de trabajo, que consistía en la selección de manera individual, de tres aspectos susceptibles de mejora y un deseo 
de la escuela que cada uno perseguía. Una vez seleccionados, cada miembro debía argumentar al resto, el porqué de su elección y categorizar cada uno de ellos atendiendo al nivel al que afectaba (personal, relacional, estructural) y al grado de control que nuestra comunidad tenía sobre él, en función de si era controlable, influenciable o estaba fuera de control.

De todo este análisis se obtuvieron diversas cuestiones (un total de veintiuna) que el GIAP consideraba fundamental analizar. De la selección obtenida observamos que existían temáticas que se relacionaban entre ellas, unas posiblemente causadas por otras y viceversa. Creamos un flujograma en el que se marcaba la relación existente entre cada variable. Gráficamente se observaba cuáles eran aquellos temas que originaban y provocaban más cambio. Por tanto, sintetizando el diagrama de flujos elaborado, se establecieron los principales focos de trabajo sobre los que debíamos intervenir. Así, llegamos a establecer como foco principal de la investigación, el cual ya se había vislumbrado como temática a tratar en la primera asamblea que se hizo, como puesta en común el día de recogida de toda la información: las relaciones personales que se establecen en el centro educativo y en el entorno, por lo que el título de nuestra investigación quedó determinado así: "Mejorar la convivencia en nuestro colegio y en el entorno".

\section{Figura 2}

Flujograma desarrollado por el GIAP a partir de las problemáticas señaladas en los papelógrafos por toda la comunidad del CEIP La Parra

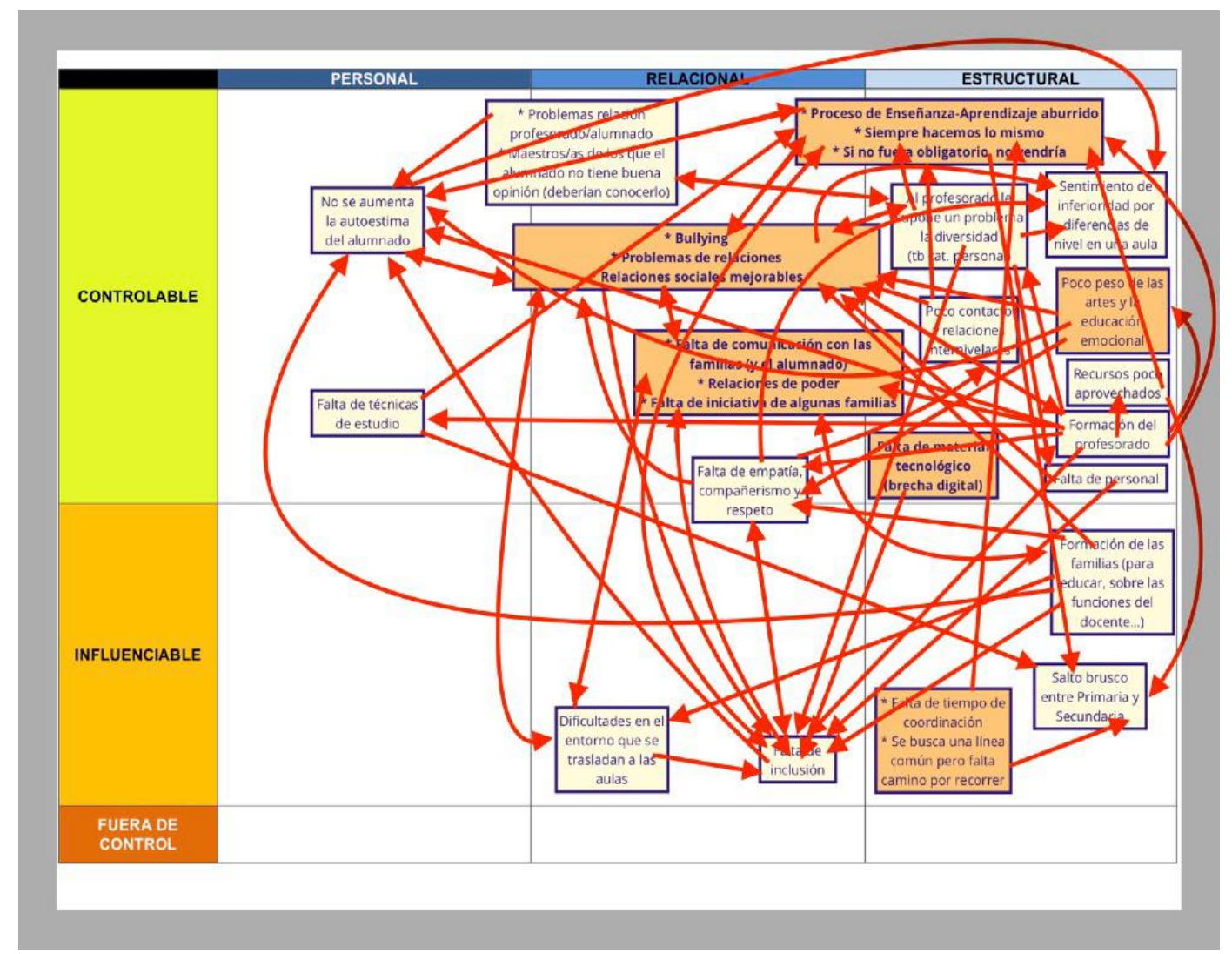

Tras establecer una temática de investigación, el GIAP planteaba la necesidad de volver a preguntar a la comunidad educativa sobre qué entendían por convivencia, cómo valoraban que eran las relaciones en su entorno, cómo pensaban que podían mejorarse y cuáles consideraban factores determinantes que impedían el desarrollo de una buena convivencia en todos los 
ambientes. El objetivo era trabajar la convivencia entre todos y todas, convencidos que esas reflexiones eran el punto de partida del trabajo hacia la mejora de la misma.

Para ello decidimos establecer una serie de herramientas que nos sirvieron para recoger dicha información: entrevistas, sociogramas y observaciones participantes. La entrevista resultó ser una estrategia de recogida de información muy útil, ya que nos permitiría conocer el pensamiento y los sentimientos de las personas de la comunidad sobre la convivencia en nuestro colegio y en el entorno, ya que las entrevistas son una herramienta que nos permite ahondar en las creencias y opiniones de las personas, dándoles libertad para poder expresar sus percepciones más subjetivas. Asimismo, la observación participante permitió investigar las prácticas y los comportamientos de otras personas, siendo especialmente eficaz para explorar las relaciones personales que se establecen en el aula entre el alumnado y entre el alumnado y el profesorado (Fàbegres et al., 2016). Por último, el sociograma es una herramienta que nos ha permitido conocer y analizar las interacciones grupales (Urbina Hurtado et al., 2018).

\section{Figura 3}

\section{Entrevistas}

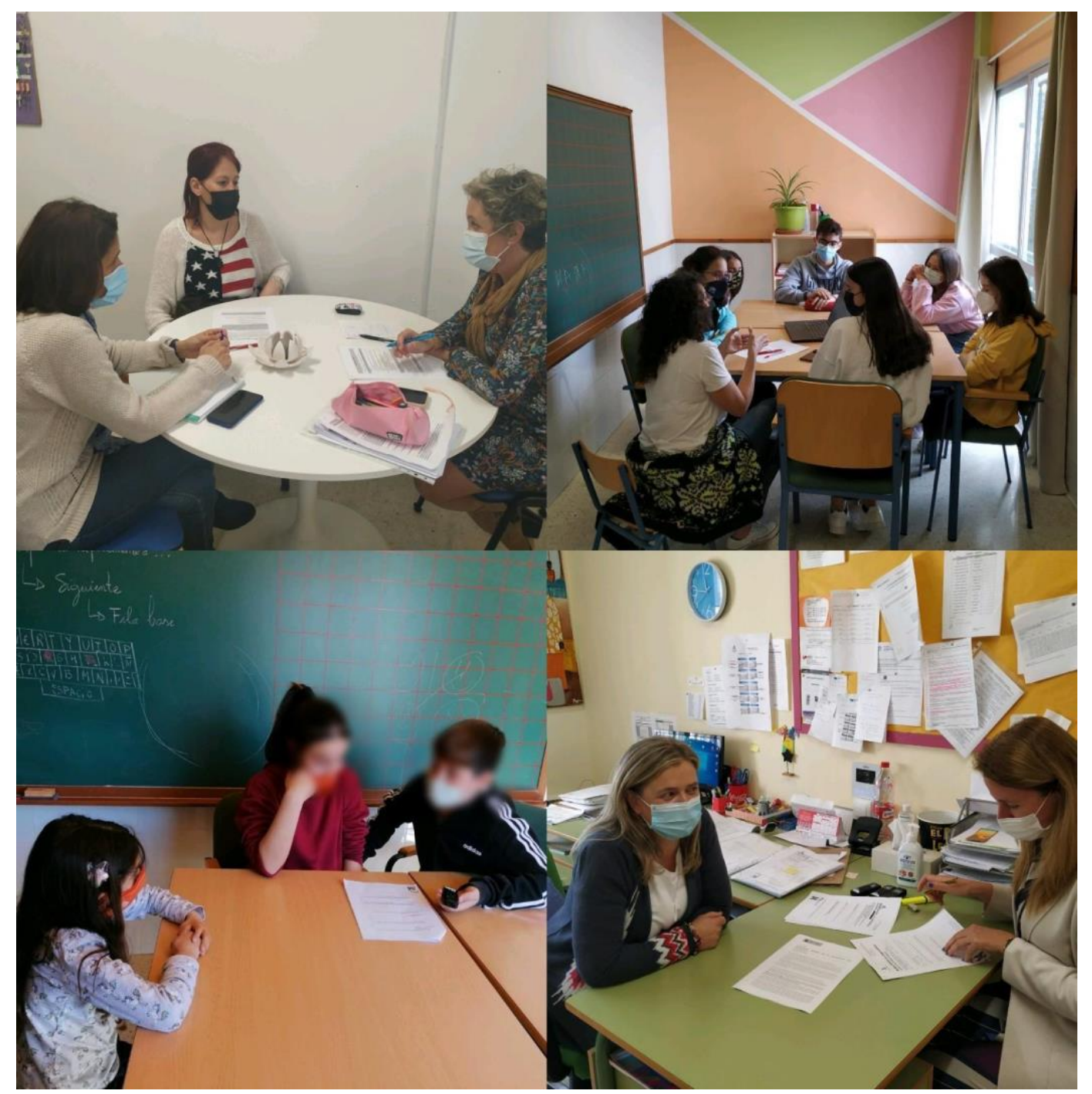

Las entrevistas se elaboraron entre todos los miembros del GIAP, creándose una batería de preguntas que servirían para provocar un momento de diálogo, encuentro y reflexión sobre la convivencia. En todo momento, se explicó que las preguntas serían abiertas suponiendo un apoyo 
y un motor para comenzar a dialogar, y no un documento cerrado que impidiese divagar al entrevistado por las cuestiones y experiencias que les parecieran más interesantes de expresar.

Asimismo, se estableció un documento de recogida de información para la observación participante que facilitase al alumnado y al profesorado sintetizar los datos que viesen relevantes sobre convivencia. Una vez elaborados todos los instrumentos de recogida de información, pensamos en la divulgación de un video explicativo que sirviera para motivar a la comunidad educativa para que participara de nuevo, con aportaciones, a las cuestiones que se le planteaban.

Toda la comunidad educativa estaba comprometida con la recogida de información. Las herramientas que utilizamos fueron instrumentos que nos aportaron muchos datos al grupo GIAP, no sólo por el contenido recogido en los mismos, sino porque todo el proceso era un continuo feedback de información. Habíamos conseguido que todos los sectores de nuestra comunidad estuvieran pensando, en un mismo periodo de tiempo, sobre convivencia.

El siguiente paso era el análisis de todos los datos. Para ello, decidimos que al igual que en la recogida, debía de realizarse entre todos los sectores de la comunidad educativa para sacar las conclusiones y extraer los aspectos más relevantes. Ambas fases (recogida y análisis) han sido procesos muy interesantes y gratificantes. El alumnado ha aprendido y disfrutado mucho realizando entrevistas a sus compañeros y así lo manifestaron a algunos de sus maestros y maestras "maestra, realizando las entrevistas a mis compañeros me siento alguien importante", o bien manifestando que "el colegio es una casa divertida"

De las propias entrevistas realizadas entre sí por el alumnado ya se dilucidaron propuestas muy interesantes tales como, "para mejorar la convivencia se podrían realizar charlas en la clase donde expliquen que no pasa nada al meterse en un problema para ayudar", "hacer juegos donde pongan a los compañeras y compañeros que no se lleven bien para que se vayan haciendo amigos” e incluso algunos entrevistadores ya dejaron reflejada su opinión sobre esta herramienta utilizada en esta fase de la investigación "la convivencia, con estas entrevistas, va a mejorar"

Las familias y otras personas de la comunidad también han manifestado sentir que las entrevistas les han ayudado a comprender la realidad. Una madre integrante del GIAP, comentaba que su entrevistada, "días después de la entrevista seguía reflexionando sobre la misma”, expresando lo significativo que le había resultado la experiencia de hablar con otra madre sobre la educación de su hijo o hija. Otra compañera del GIAP, tras una entrevista, expresaba "me ha agradado mucho la experiencia y la persona entrevistada, desde el primer momento ha entendido la finalidad de la entrevista y ha sido muy sincera, cosa que se la he agradecido un montón”.

En el análisis de la información recogimos muchos datos significativos que nos arrojaron luz sobre cómo es la convivencia en nuestro colegio y en el entorno social del mismo. Han emergido distintas preocupaciones que tienen que ver con las relaciones personales.

Por un lado, las que se establecen a través de las nuevas tecnologías o las redes sociales ya que como expresaba una madre en la entrevista "ahora las relaciones son distintas, hay más tecnología”. Por otro lado, también preocupan los vínculos que se establecen en el aula entre el alumnado, que no siempre son positivos, como explica un alumno en una entrevista, "no me esfuerzo, prefiero no perder tiempo con gente que no me cae bien”. Y por último, los lazos que se establecen durante el recreo entre el alumnado, y cómo todas estas formas de relación influyen en el aprendizaje de las niñas y de los niños, "algunas veces hay algunos compañeros que cuando quiero jugar con ellos me rechazan" (entrevista realizada de un alumno a otro alumno de Primaria) 
Otro aspecto relevante de la investigación tiene que ver con la interacción que se produce entre las personas del entorno "Las relaciones en el colegio son como las de siempre, van al colegio, toman la clase y vuelven a casa... Pasan demasiado tiempo allí encerrados y se pierden la vida del pueblo que transcurre fuera... Hay mucha tecnología pero se olvidan del contacto con otras personas", expresaba una madre de Primaria durante la entrevista que le hacía otra madre del GIAP, tomando conciencia de que el colegio no puede verse como un entorno aparte para el alumnado y de la importancia que tiene la colaboración entre escuela, familia y entorno social.

Algunas familias demandaban al colegio una función formativa también con las familias, creen que el centro debe "dar herramientas a las familias para una crianza más respetuosa, pues en casos en los que este tipo de crianza está presente, la educación de los hijos es mejor" (entrevista a una madre de Primaria) o "intentar implicar más a los hombres" (entrevista a una madre de Primaria).

Con toda la información se elaboró un único documento donde se plasmaban las aportaciones y conclusiones más relevantes de las entrevistas y observaciones llevadas a cabo, extrayendo de toda la información analizada tres categorías principales sobre las que se debía trabajar. La primera categoría que emergió estaba relacionada con el uso de las redes sociales. Se había detectado que había una preocupación por parte de las familias y el profesorado, debido a las relaciones que se establecían a través de estos medios. Un uso indebido de las mismas, estaba ocasionando continuos conflictos entre los iguales, que derivaban de manera directa, en las relaciones que se dan en el centro. Se evidenciaba que se estaban normalizando ciertas maneras de proceder a través de estas nuevas tecnologías que empeoraban las relaciones entre ellos. Era imprescindible por tanto, concienciar y trabajar sobre este tema implicando a toda la comunidad, sobre la importancia del buen uso de las redes sociales para obtener una mejor convivencia.

La segunda categoría que surgió, tenía relación con las metodologías. Se evidenciaba cómo la aplicación de un tipo de metodología u otra en el entorno escolar, se vinculaba íntimamente con las relaciones que se establecía entre el alumnado; y entre el alumnado y el profesorado, "ayudando se aprende" (entrevista realizada entre alumnado de Secundaria). El alumnado demandaba una metodología más activa, participativa y cooperativa. "Cuando terminamos las tareas, hay tiempo libre para jugar, dibujar... pero a mí me gustaría aprender siempre con juegos, talleres, ordenadores,...me gustaría aprender divirtiéndome, con menos fichas y menos exámenes" (entrevista realizada entre alumnado de Primaria ). Ponían el foco en que se puede aprender divirtiéndose siendo dicho aprendizaje más duradero en el tiempo que cuando se hace de manera más tradicional.

El profesorado en esta línea reflexionaba sobre su práctica docente opinando que "hay poca convivencia cuando la clase es magistral y más convivencia cuanto más participa el alumnado y más tienen que cooperar" (reflexión de observación participante de una docente). Las familias, sin embargo, son el sector de la comunidad educativa que más se resiste al cambio metodológico. Se ha percibido que hay una doble concepción con respecto a esta temática, quienes apoyan un cambio metodológico y los que se niegan a dicho cambio y se inclinan por una metodología más tradicional parecida a la que ellos y ellas tuvieron cuando eran escolares, convencidos que así sus hijos e hijas aprenden más. Otros miembros de la comunidad expresaban "Estoy de acuerdo en que influye mucho la metodología del profesorado. Entiendo que si el profesorado da clases aburridas los alumnos solo memorizan para los exámenes, mientras que si las clases son didácticas y divertidas lo que aprenden no lo olvidaran" (entrevista realizada a un miembro de la comunidad) 
Y la última categoría venía establecida por la importancia que tiene el horario del recreo para el alumnado, ya que constituye un espacio educativo muy rico para aprender y establecer relaciones sociales entre iguales. Se trata, por tanto, de un momento del día donde se aglutinan muchas experiencias, se evidencian los distintos tipos de relaciones que se dan entre ellos y se ponen en juego todas las habilidades sociales y de relación que el alumno tiene adquirida. Repensar el recreo como espacio educativo se presenta, entonces, como un gran reto que puede mejorar enormemente el bienestar del alumnado en desventaja y una gran mejora en la convivencia del centro. "En el recreo es donde se producen la mayoría de los problemas de convivencia" (Entrevista realizada entre alumnado). "Hay alumnado que está solo" (reflexión de observación participante de una docente). "El alumnado no tiene alternativas de juego o actividades" (Reflexión de observación participante de una docente).

\subsection{Jornada de devolución creativa}

Establecidas ya las tres categorías sobre las que debíamos trabajar: redes sociales, relaciones y cultura; la metodología como formas de relación; y el recreo como espacio educativo, consideramos que era necesario devolver esta información a toda la comunidad educativa. Primero porque las conclusiones a las que habíamos llegado venían derivadas de las distintas voces y era justo que conocieran los resultados que se habían obtenido; y segundo porque, en nuestro deseo de transformación y cambio para nuestra escuela, era necesario la implicación de todos los sectores para que dicho cambio se produjera. Era imprescindible, por tanto, escuchar de nuevo a todas las partes para que aportaran cómo podíamos provocar ese cambio.

El grupo GIAP comenzó entonces a pensar cómo podíamos organizar un encuentro donde estuviera toda la Comunidad Educativa. En las Jornadas de devolución creativa la comunidad debía verse representada en todo lo que contáramos y debían provocar una reflexión y debate conjunto, dinamizando a las personas, para seguir avanzando en el análisis que diera lugar a la construcción de la escuela que queremos.

Nos planteamos ahora cómo hacer la devolución. Después de tener en cuenta varias posibilidades, fue el alumnado principalmente, quien apostó por la creación de videos que sirvieran de presentación a toda la comunidad de lo que estábamos realizando y de introducción a los tres bloques sobre los que queríamos intervenir. El montaje de dichos vídeos, así como el contenido de los mismos fue creación del alumnado. Establecimos entonces cómo íbamos a realizar dicha jornada de devolución, los medios y las plataformas que utilizaríamos y el modo de invitar a la comunidad para que fuera motivador y asegurara la asistencia de un gran número de personas. La organización de dicha jornada tenía un doble objetivo. Por un lado devolver la información, y por otro lado establecer un encuentro donde se recogieran las aportaciones.

\subsection{Diseño del Plan de Acción Integral (PAI): Elaboración de propuestas}

La jornada de devolución creativa se organizó de manera que se crearan espacios donde todas las personas participantes pudieran intervenir, dialogando y proponiendo ideas de mejora. De ese encuentro se recogieron muchas propuestas que se unieron a las que ya había. Con todas ellas, en el GIAP se elaboró un Plan de Acción Integral (PAI) para llevar dichas propuestas a la práctica.

En cuanto a las redes sociales, relaciones y cultura se plantean las siguientes acciones:

- Creación de un área curricular de diseño propio "Educación en Redes" para el alumnado de $4^{\circ}, 5^{\circ}$ y $6^{\circ}$ de Educación Primaria, con la finalidad de educar a alumnado y familias en el uso responsable de las Redes Sociales y las Nuevas Tecnologías, así como educar en las competencias digitales. 
- Charlas para la Convivencia y Mejora de la Seguridad Escolar en los Centros Educativos y su Entorno"

Relativo a las metodologías como formas de relación las intervenciones a llevar a cabo serían:

- Fomento del aprendizaje cooperativo en las aulas.

- Uso de distintas herramientas digitales para la evaluación del alumnado.

- Organización de espacios educativos distintos al aula como, por ejemplo, utilizar el patio como lugar de aprendizaje.

- Formación docente en metodologías inclusivas como el Diseño Universal del Aprendizaje.

\section{Figura 4}

\section{Cambios metodológicos en el aula}
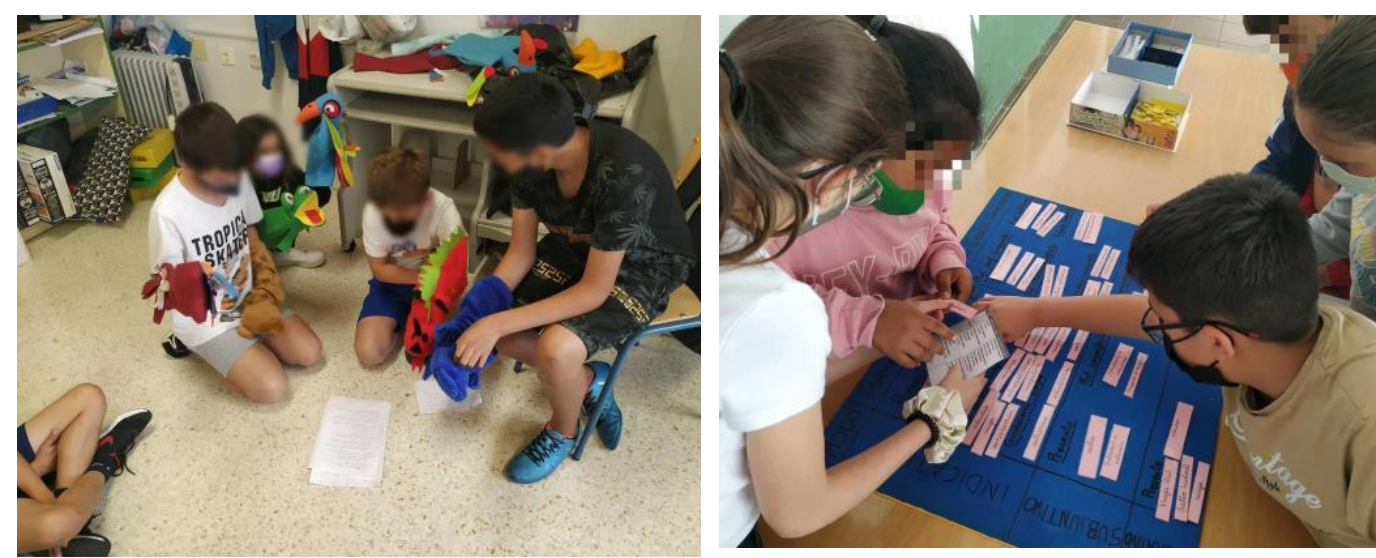

En relación a las propuestas del recreo como espacio educativo:

- Materiales de juegos para el recreo.

- Música en el recreo

- Cambio de espacios en el patio de los grupos burbuja para que no siempre estén en la misma zona.

- Creación de espacios verdes en el patio: jardín vertical y un huerto en macetas.

- Mesas con bancos en el patio para que el alumnado pueda leer o jugar a juegos de mesa en el porche cubierto.

Todas estas propuestas de acción tienen por finalidad que sean posibles nuevas formas de relación entre el alumnado, y entre el alumnado y el profesorado, formas de relación que mejoren la convivencia en el colegio y en su entorno. 


\section{Figura 5}

\section{Juegos en el recreo}
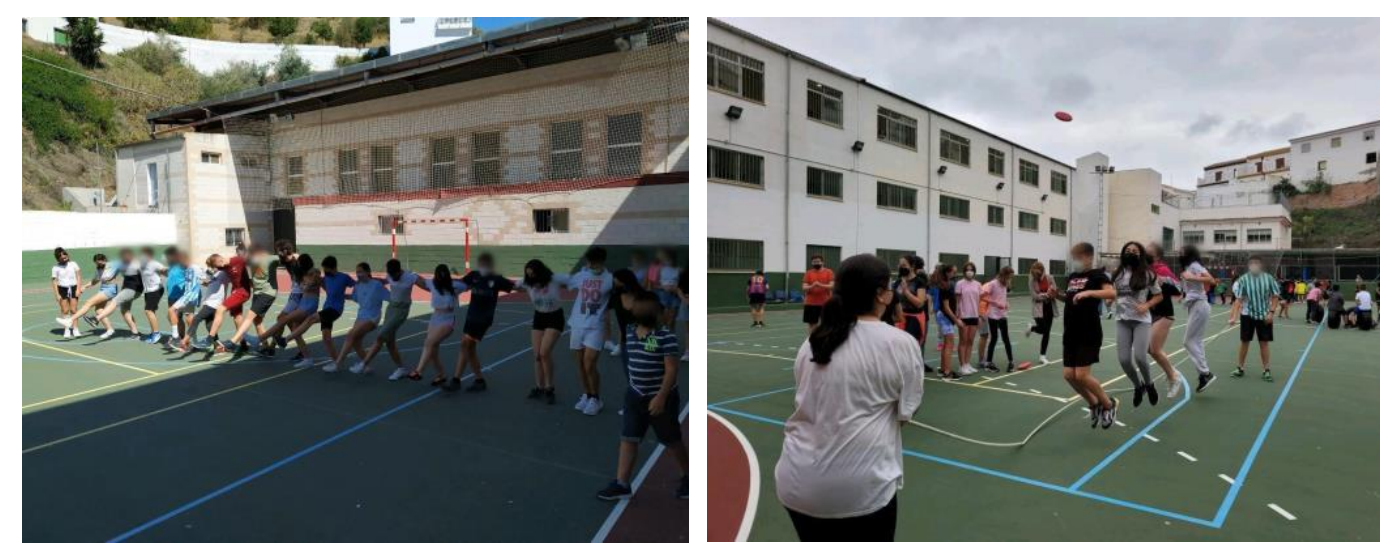

\subsection{Evaluación del ciclo}

Para evaluar el Plan de Acción Integral se llevaron a cabo tres instrumentos concretos de evaluación, en ellos se contaba con la participación del alumnado, del profesorado y de los componentes del GIAP.

El primer instrumento utilizado fue con el objetivo de dar voz a los niños, niñas, y profesorado en un mismo escenario. El alumnado es una parte importante de la evaluación ya que pueden "identificar las barreras, así como las fortalezas en las prácticas docentes con el objetivo de que todos los alumnos y alumnas, sin exclusiones, aprendan y participen” (Sandoval et al., 2020, p. 23). Para ello, se realizaron asambleas en cada aula del centro educativo con el fin de poder debatir sobre las acciones desarrolladas. La finalidad de dichas asambleas consistió en analizar y reflexionar con sus integrantes todos los cambios producidos en el centro y recoger las nuevas propuestas de actuación.

El segundo instrumento realizado fue la creación de un espacio donde el alumnado que voluntariamente quisiera, pudiera dar su opinión o su valoración de las distintas intervenciones realizadas. Para ello se construyó nuestro "fotomatón", donde el alumnado de forma privada expresaba en una grabación todas las aportaciones, críticas, propuestas que querían compartir sobre la experiencia vivida.

Y el tercer y último instrumento se llevó a cabo por el GIAP realizando varias sesiones dedicadas a la autoevaluación del trabajo realizado por parte de las personas integrantes del grupo, con la finalidad de mejorar el proceso. Para ello, se realizó un análisis DAFO donde se recogían las dificultades, fortalezas, amenazas y oportunidades.

Las herramientas participativas empleadas en esta fase de evaluación fueron: las técnicas de observación, diálogo, reuniones con la comunidad, asambleas y lluvia de ideas.

Entre las dificultades detectamos que mayoritariamente todos los integrantes consideran que ha habido muy poco tiempo para la intervención, además de otras encontradas como la poca diferencia en la distribución de responsabilidades. Algunas de las fortalezas detectadas, son la implicación y participación de toda la comunidad educativa en el proyecto, y la producción de los cambios en tiempo real. Además, las voces de toda la comunidad han tomado su peso en este cambio, ya que las propuestas realizadas han sido llevadas a cabo. Asimismo, se ha producido un 
cambio profundo en nuestra cultura, "orientándola hacia una cultura más colaborativa" (DíezGutiérrez, 2020, p. 123).

\section{Figura 6}

\section{Jornada de evaluación del GIAP}

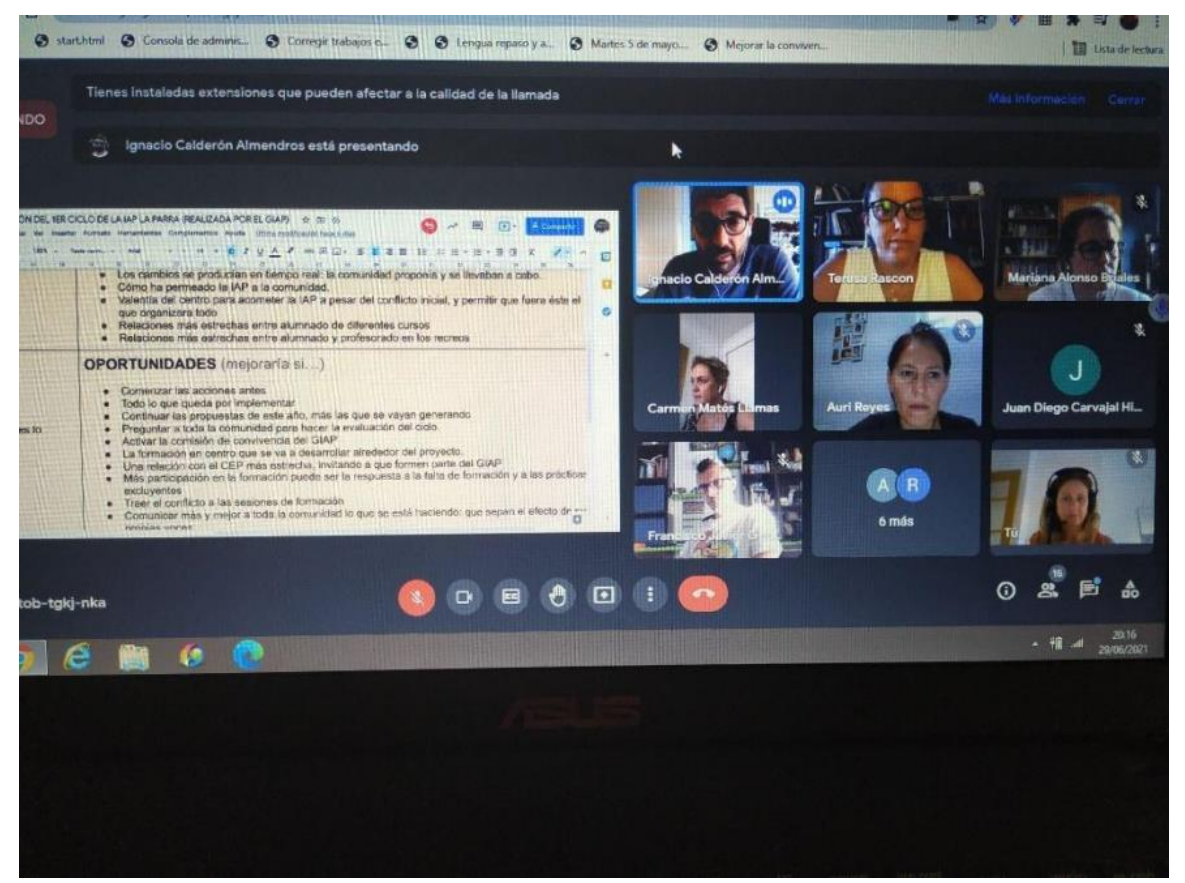

Entre los peligros encontrados se ha evidenciado el desgaste que han sufrido las personas que mayores responsabilidades asumían, así como la falta de formación del profesorado. Como oportunidades de mejora destacar que se ha tomado conciencia, por otro lado, que la investigación mejoraría si se continuara con una formación en el centro en la que participara todo el claustro de profesorado.

\section{Resultados}

La IAP ha generado vínculos que no existían previamente, y esto ya tiene un sentido en sí mismo, es decir, el mismo proceso de investigación ya ha supuesto un cambio en las relaciones que se establecían antes en el colegio y en la Comunidad. Las evidencias de esta experiencia de intervención recogidas en las asambleas realizadas y en el fotomatón, muestran una mejora del clima escolar, la modificación de algunas prácticas pedagógicas, así como el posicionamiento del alumnado como protagonista del proceso de implementación de esta investigación. Se puede afirmar que la mejora de la convivencia no se da a partir de la implementación de normas, sino que es necesario impulsar y llevar a cabo acciones y metodologías que transformen las prácticas escolares. "Me ha encantado esto que hemos hecho de las entrevistas para hacer nuestro colegio mejor (Voz de un alumno en la asamblea de evaluación).

Los cambios en el recreo van poco a poco transformándolo en un espacio más educativo:

Antes no teníamos la oportunidad de jugar con todos porque a veces eran muy brutos pero ahora como tenemos los materiales, los niños están cambiando un poquito y están empezando a jugar más tranquilos y ahora tenemos la oportunidad de jugar con todos. (Voz de una alumna en la asamblea de evaluación) 
Estas variaciones han permitido, a su vez, que el alumnado que estaba solo o aburrido tuviera otras opciones de juego. "Los cambios en el patio ha sido muy buena idea porque usamos materiales y nos divertimos más, lo de cambiar cada semana de patio también me ha parecido muy bien" (Voz de una alumna en la asamblea de evaluación). Aunque no han estado exentos de problemas que han sido oportunidades de aprendizaje. "En nuestra aula tuvimos un problema porque no sabíamos compartir, pero lo solucionamos pensando que lo mejor era jugar todos juntos compartiendo los materiales" (Voz de un alumno en la asamblea de evaluación).

Con respecto a la metodología el alumnado ha expresado que les gusta aprender de una forma más participativa porque "la nota del examen no importa, importa aprender" (Voz de un alumno en la asamblea de evaluación), y que "si una persona no puede, hay que enseñar a la persona de otra manera para que pueda aprenderlo" (Voz de un alumno en la asamblea de evaluación).

Nosotros desde primero de primaria hemos tenido la metodología de estudio de libros, estudiar, examen. To me he adaptado a eso perfectamente y a lo que sea me adapto, ¿̇pero qué pasa?, que hay veces que con eso, tú lo que haces es que estudias dos días antes, luego lo pones en el examen, y ya se te ha olvidado. To no me acuerdo de lo que dimos de Geografía e Historia en el primer trimestre y saqué un 9,65. $\Upsilon$ los demás exámenes los tengo igual y tampoco me acuerdo. Hay gente que a lo mejor se aburre o no tiene la misma capacidad de adaptación a todo. A lo mejor si se trabaja de otra forma (...), lo sigues recordando más tiempo. (Voz de una alumna en la asamblea de evaluación)

Se ha evidenciado que el aprendizaje cooperativo para el alumnado "es mejor porque así nos ayudamos" (voz de un alumno en la asamblea de evaluación) porque aprenden a relacionarse de otra manera. "Pienso que se aprende más trabajando en equipo porque ganas habilidad para trabajar con alguien" (Voz de un alumno en la asamblea de evaluación).

Una alumna explica que,

\begin{abstract}
Hemos cambiado mucho la forma de estudiar porque antes siempre cogíamos los libros y ahora cogemos los ordenadores y en vez de hacer exámenes repasamos con otras cosas (...) Ahora se hacen más Kahoot y más juegos. Creo que la metodología ha cambiado mucho y me parece que es un cambio a mejor porque se aprende más jugando que con libros y mandándonos ejercicios, porque si la clase es divertida se te queda en la mente. (Voz de una alumna en la asamblea de evaluación)
\end{abstract}

sobre las redes sociales y las nuevas tecnologías, el alumnado explica que les parece muy importante que el curso que viene el alumnado de $4^{\circ}, 5^{\circ}$ y $6^{\circ}$ tengan una asignatura donde puedan aprender sobre los riesgos y los beneficios de las tecnologías, y que también deberían aprender las familias

la escuela debe educar en las redes a las familias y al alumnado (...) los niños y niñas con 6 y 7 añitos ya tienen redes sociales, Facebook, Instagram, cuenta de Youtube, Whats App propio, (...), sí, es importante que las niñas y niños, como lo usan ya cada vez más pronto, lo aprendan antes de llegar a tener dificultades. (Voz de una maestra en la asamblea de evaluación)

Asimismo, opinan que las charlas que se imparten en el colegio, como la del Plan Director, son importantes porque les informan de los riesgos que tiene usar las Redes Sociales que antes no conocían:

De las charlas que hemos hecho hoy he aprendido mucho y me he dado cuenta que puedo hacer daño a otra persona. (Voz de un alumno en la asamblea de evaluación)

\title{
5. Discusión y conclusiones
}

La Investigación Acción Participativa nos ha dado la posibilidad de ser protagonistas de la construcción de la escuela que queremos, y en el proceso, nos hemos dado cuenta de que generar 
conocimiento y reflexiones colectivas enriquece los proyectos. Nos ha permitido conocer con más profundidad nuestro contexto educativo, y el diálogo entre todos los miembros de la Comunidad Educativa, ha desarrollado el pensamiento crítico y la conciencia de la realidad que vivimos. El crear un espacio donde debatir sobre temas que nos preocupan con un grupo de personas integrantes de nuestra comunidad educativa, nos hace crecer como personas y profesionales en este sector de la educación. Prueba de ello es que la Investigación Acción Participativa está considerada como una de las técnicas más idóneas para alcanzar objetivos relacionados con el diseño e implementación de experiencias de mejora en los centros educativos (Colás et al., 2009). Compartimos con Valderrama-Hernandez y Solís-Espallargás (2015, p. 58) que "la comunidad conoce y reconoce su territorio de forma diferente a como lo hace la institución y que la combinación entre estos tipos de saberes permitirá un mayor acercamiento a la realidad y una mejor toma de decisiones sobre el territorio".

Mejorar la convivencia en nuestro colegio y en nuestro entorno es hacer nuestra escuela más inclusiva, como explica Echeita y otros (2019, p. 73), "la educación inclusiva sólo avanzará significativamente si, y sólo sí, se pone en marcha una transformación profunda de los sistemas educativos excluyentes que ahora tenemos". Así, "las prácticas docentes que fomentan una pedagogía inclusiva deben sustentarse en una evaluación que implique a todos los agentes que participan en los procesos de enseñanza-aprendizaje" (Sanahuja et al., 2020, p. 1). Hay muchas experiencias, como las que describen Tomé y Manzano-García (2016) que ponen de manifiesto la capacidad transformadora de la investigación-acción en los contextos educativos.

Siguiendo a Ainscow (2017, p. 44), "hacerse más inclusivo es una cuestión de reflexión y diálogo, de revisión y redefinición de la práctica, y de intentar desarrollar una cultura más inclusiva”. A través de la Investigación-Acción Participativa fomentamos la reflexión, el diálogo y la revisión de nuestras prácticas docentes para construir la cultura de la diversidad en nuestra Comunidad.

Como conclusión nos gustaría destacar que este camino que hemos emprendido hacia la transformación y la cualificación de nuestra escuela y de su entorno no acaba aquí, este solo es un paso más de un camino que no acaba nunca, el de la reflexión y la mejora constante. Ilusionadas por seguir construyendo y transformando entre todos y todas.

\section{Referencias}

Ainscow, M. (2017). Haciendo que las escuelas sean más inclusivas: Lecciones a partir del análisis de la investigación internacional. Revista de Educación Inclusiva, 5(1), 39-49.

Calderón Almendros, I. (2019a). Entrevista a Ignacio Calderón Almendros, realizada por Jaume Francesch i Subirana. Revista Educar y Orientar, 11, 18-21.

Calderón Almendros, I. (2019b, 3 de diciembre). Seguimos excluyendo. El Diario de la Educación. https://eldiariodelaeducacion.com/2019/12/03/seguimos-excluyendo/

Colás, M. P., Buendía, L. y Hernández Pina, F. (2009). Competencias científicas para la realización de una tesis doctoral. Davinci.

Colmenares Escalona, A. M. (2012). Investigación-acción participativa: Una metodología integradora del conocimiento y la acción. Voces y Silencios, 3(1), 102-115. https://doi.org/10.18175/vys3.1.2012.07

Díez-Gutiérrez, E. J. (2020). Otra investigación educativa posible: Investigación-acción participativa dialógica e inclusiva. Márgenes, Revista de Educación de la Universidad de Málaga, 1(1), 115-128. https://doi.org/10.24310/mgnmar.v1i1.7154 
Díez-Palomar, J. y Flecha García, R. (2010). Comunidades de aprendizaje: un proyecto de transformación social y educativa. Revista Interuniversitaria de Formación del Profesorado, 24, 19-30.

Delors, J. (1996). La educación o la utopía necesaria. En J. Delors, I. Al Mutfi, R. Carneiro, F. Chung, B. Geremek, W. Gorham, A. Kornhauser, M. Manley, M. Padrón Quero, M. A. Savané, K. Singh, R. Stavenhagen, M. Won Suhr y Z. Nanzhao, La educación encierra un tesoro (pp. 13-35). Santillana.

Echeita, G. (2019). Educación inclusiva: El sueño de una noche de verano. Octaedro.

Echeita, G., Simón, C., Sandoval, M., Calero, C., Nuñez Gutiérrez de San Miguel, B., Sotto Esteban, P., Pérez García, M. M., García de la Torre, A. B. (2016). Transformando la "gramática escolar" para ser más inclusivos: La experiencia de tres centros educativos. Contextos Educativos, 19, 7-24. https://doi.org/10.18172/con.2784

Elliott, J. (1993). El cambio educativo desde la investigación acción. Morata.

Freire, P. (1994). Pedagogía de la esperanza. Un reencuentro con la Pedagogía del oprimido. Siglo XXI Editores.

Freire, P. (1997). Pedagogía para la autonomía: Saberes necesarios para la práctica educativa. Siglo XXI.

Tomé, M. y Manzano-García, B. (2016). La investigación en la práctica docente. Ediciones Nobel.

Kemmis, S. y Mc'Taggart, R. (1988). Cómo planificar la investigación-acción. Laertes.

Latorre, A. (2003). La investigación acción: conocer y cambiar la práctica educativa. Graó.

López Melero, M. (2008). ¿Es posible construir una escuela sin exclusiones? Revista Brasileña de Educación Especial, 14(1), 3-20. https://doi.org/10.1590/S1413-65382008000100002

Machado, A. (1991). Poesías completas. Espasa Calpe.

Melero, N. (2011). El paradigma crítico y los aportes de la investigación acción participativa en la transformación de la realidad social: Un análisis desde las ciencias sociales. Cuestiones Pedagógicas, 21, 339-355.

Pereda, C. y Prada, M. Á. de (2014). Investigación-acción participativa y perspectiva dialéctica. Arxius de Sociologia, 31, 57-68.

Sanahuja, A., Moliner, L. y Benet, A. (2020). Análisis de prácticas inclusivas de aula desde la investigaciónacción participativa. Reflexiones de una comunidad educativa. Revista Internacional de Educación para la Justicia Social, 9(1), 125-143. https://doi.org/10.15366/riejs2020.9.1.006

Sandoval, M., Simón, C. y Echeita, G. (2020). ¿Qué me ayuda a aprender y participar? Herramientas para recoger las voces de los estudiantes. Revista de Educación Inclusiva, 13(1), 12-27.

Urbina Hurtado, C., López Leiva, V. y Cárdenas Villalobos, J. P. (2018). El uso de sociogramas en la escuela para la mejora de la convivencia. Perfiles Educativos, 160(6), 83-100. https://doi.org/10.22201/iisue.24486167e.2018.160.58398

Valderrama Hernández, R. y Solís Espallargas, C. (2015). Investigación acción participativa como estrategia de transformación social y ambiental. Investigación en la Escuela, 86, 49-59.

Zeichner, K. M. (2010). La formación del profesorado y la lucha por la justicia social. Morata.

\section{Breve CV de las autoras}

\section{Ma del Mar Herrera Fernández}

Maestra de Educación Infantil. Jefa de estudios del CEIP La Parra desde el curso 2011/2012. Coordinadora del proyecto de innovación/investigación en colaboración con la Universidad de 
Málaga "Desarrollo de Habilidades Metalingüísticas en Educación Infantil en el CEIP La Parra en Almáchar”. Ha realizado diversas ponencias en relación a las Comunidades de Aprendizaje así como Jornadas de exposición de proyectos y experiencias en Educación Infantil. Integrante del Grupo de Investigación Acción Participativa de la Comunidad Educativa de La Parra (Almáchar) en el marco de la Investigación "Narrativas emergentes sobre la escuela inclusiva desde el Modelo Social de la Discapacidad. Resistencia, resiliencia y cambio social”, de la Universidad de Málaga. Referencia: RTI2018-099218-A-100. Email: meryherrera76@gmail.com

ORCID ID: https://orcid.org/0000-0001-5781-9584

\section{Carmen Matés Llamas}

Maestra de Educación Primaria, Educación Musical y Pedagogía Terapéutica. Licenciada en Psicopedagogía por la Universidad de Málaga. Funcionaria del Cuerpo de Maestros desde septiembre de 2001. Directora del CEIP La Parra desde el curso 2011/2012. Ha realizado diversas ponencias para el Centro de Educación Permanente de profesores de la Axarquía y Málaga en relación a las Comunidades de Aprendizaje. Integrante del Grupo de Investigación Acción Participativa de la Comunidad Educativa de La Parra (Almáchar) en el marco de la Investigación "Narrativas emergentes sobre la escuela inclusiva desde el Modelo Social de la Discapacidad. Resistencia, resiliencia y cambio social”, de la universidad de Málaga. Referencia: RTI2018-099218-A-100. Email: matescarmen@gmail.com

ORCID ID: https://orcid.org/OOOO-0002-4767-8164

\section{Diana Farzaneh Peña}

Maestra e investigadora de primaria del Proyecto Roma y de la escuela pública. Especialista en Pedagogía Inclusiva. Máster en Políticas y Prácticas de Innovación Educativa por la Universidad de Málaga. Integrante del Grupo de Investigación Cultura de la Diversidad y Escuela HUM-246. Integrante del Grupo de Investigación Acción Cooperativa Formativa de Familias del Proyecto Roma. Integrante del Grupo de Investigación Acción Participativa de la Comunidad Educativa de La Parra (Almáchar). Coordinadora de Orientación, Coordinadora del Proyecto Escuela: Espacio de Paz y Coordinadora del Plan de Igualdad en el CEIP La Parra en Almáchar. Ha realizado varias publicaciones en revistas como UNO, RIFOP e IJNE, y algunas ponencias en diferentes jornadas educativas y en la Universidad de Málaga. Email: dianafarzaneh79@gmail.com

ORCID ID: https://orcid.org/0000-0001-6070-0082

\section{Sandra Barrado Fernández}

Graduada en Educación Social por la Universidad de Málaga y Técnica Superior en Integración Social. Título de Máster Universitario en Profesorado de Educación Secundaria Obligatoria y Bachillerato, Formación Profesional y Enseñanza de Idiomas en la especialidad de Formación y Orientación Laboral. Ha sido becaria de colaboración en el departamento de Teoría e Historia de la Educación y Métodos de Investigación y Diagnóstico en Educación de la Universidad de Málaga. Actualmente es Personal Técnico de Integración Social en CEIP La Parra (Almáchar, Málaga) y cursa el Máster Universitario en Cambio Social y Profesiones Educativas en la Universidad de Málaga. Email: sandra.fdez.92@gmail.com

ORCID ID: https://orcid.org/o000-0001-5175-3932 